Article

\title{
On Generalized Closed Sets and Generalized Pre-Closed Sets in Neutrosophic Topological Spaces
}

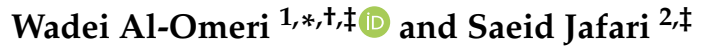 \\ 1 Department of Mathematics, Al-Balqa Applied University, Salt 19117, Jordan \\ 2 Department of Mathematics, College of Vestsjaelland South, Herrestraede 11, 4200 Slagelse, Denmark; \\ jafaripersia@gmail.com \\ * Correspondence: wadeialomeri@bau.edu.jo; Tel.: +962-77-6690-543 \\ + Current address: Department of Mathematics, Al-Balqa Applied University, Salt 19117, Jordan. \\ $\ddagger$ These authors contributed equally to this work.
}

Received: 17 November 2018; Accepted: 13 December 2018; Published: 20 December 2018

check for updates

\begin{abstract}
In this paper, the concept of generalized neutrosophic pre-closed sets and generalized neutrosophic pre-open sets are introduced. We also study relations and various properties between the other existing neutrosophic open and closed sets. In addition, we discuss some applications of generalized neutrosophic pre-closed sets, namely neutrosophic $p T_{\frac{1}{2}}$ space and neutrosophic $g p T_{\frac{1}{2}}$ space. The concepts of generalized neutrosophic connected spaces, generalized neutrosophic compact spaces and generalized neutrosophic extremally disconnected spaces are established. Some interesting properties are investigated in addition to giving some examples.
\end{abstract}

Keywords: neutrosophic topology; neutrosophic generalized topology; neutrosophic generalized pre-closed sets; neutrosophic generalized pre-open sets; neutrosophic $p T_{\frac{1}{2}}$ space; neutrosophic $g p T_{\frac{1}{2}}$ space; generalized neutrosophic compact and generalized neutrosophic compact

\section{Introduction}

Zadeh [1] introduced the notion of fuzzy sets. After that, there have been a number of generalizations of this fundamental concept. The study of fuzzy topological spaces was first initiated by Chang [2,3] in 1968. Atanassov [4] introduced the notion of intuitionistic fuzzy sets (IFs). This notion was extended to intuitionistic L-fuzzy setting by Atanassov and Stoeva [5], which currently has the name "intuitionistic L-topological spaces". Coker [6] introduced the notion of intuitionistic fuzzy topological space by using the notion of (IFs). The concept of generalized fuzzy closed set was introduced by Balasubramanian and Sundaram [7]. In various recent papers, Smarandache generalizes intuitionistic fuzzy sets and different types of sets to neutrosophic sets (NSs). On the non-standard interval, Smarandache, Peide and Lupianez defined the notion of neutrosophic topology [8-10]. In addition, Zhang et al. [11] introduced the notion of an interval neutrosophic set, which is a sample of a neutrosophic set and studied various properties.

Recently, Al-Omeri and Smarandache [12,13] introduced and studied a number of the definitions of neutrosophic closed sets, neutrosophic mapping, and obtained several preservation properties and some characterizations about neutrosophic of connectedness and neutrosophic connectedness continuity.

This paper is arranged as follows. In Section 2, we will recall some notions that will be used throughout this paper. In Section 3, we mention some notions in order to present neutrosophic generalized pre-closed sets and investigate its basic properties. In Sections 4 and 5, we study the neutrosophic generalized pre-open sets and present some of their properties. In addition, we provide an application of neutrosophic generalized pre-open sets. Finally, the concepts of generalized neutrosophic 
connected space, generalized neutrosophic compact space and generalized neutrosophic extremally disconnected spaces are introduced and established in Section 6 and some of their properties in neutrosophic topological spaces are studied.

This class of sets belongs to the important class of neutrosophic generalized open sets which is very useful not only in the deepening of our understanding of some special features of the already well-known notions of neutrosophic topology but also proves useful in neutrosophic multifunction theory in neutrosophic economy and also in neutrosophic control theory. The applications are vast and the researchers in the field are exploring these realms of research.

\section{Preliminaries}

Definition 1. Let $\mathscr{Z}$ be a non-empty set. A neutrosophic set (NS for short) $\tilde{S}$ is an object having the form $\tilde{S}=\left\{\left\langle k, \mu_{\tilde{S}}(k), \sigma_{\tilde{S}}(k), \gamma_{\tilde{S}}(k)\right\rangle: k \in \mathscr{Z}\right\}$, where $\gamma_{\tilde{S}}(k), \sigma_{\tilde{S}}(k), \mu_{\tilde{S}}(k)$, and the degree of non-membership (namely $\gamma_{\tilde{S}}(k)$ ), the degree of indeterminacy (namely $\sigma_{\tilde{S}}(k)$ ), and the degree of membership function (namely $\left.\mu_{\tilde{S}}(k)\right)$, of each element $k \in \mathscr{Z}$ to the set $\tilde{S}$, see [14].

A neutrosophic set $\tilde{S}=\left\{\left\langle k, \mu_{\tilde{S}}(k), \sigma_{\tilde{S}}(k), \gamma_{\tilde{S}}(k)\right\rangle: k \in \mathscr{Z}\right\}$ can be identified as $\left\langle\mu_{\tilde{S}}(k), \sigma_{\tilde{S}}(k), \gamma_{\tilde{S}}(k)\right\rangle$ in $\rfloor 0^{-}, 1^{+}\lfloor$on $\mathscr{Z}$.

Definition 2. Let $\tilde{S}=\left\langle\mu_{\tilde{S}}(k), \sigma_{\tilde{S}}(k), \gamma_{\tilde{S}}(k)\right\rangle$ be an NS on $\mathscr{Z}$. [15] The complement of the set $\tilde{S}(C(\tilde{S})$, for short $)$ may be defined as follows:

(i) $C(\tilde{S})=\left\{\left\langle k, 1-\mu_{\tilde{S}}(k), 1-\gamma_{\tilde{S}}(k)\right\rangle: k \in \mathscr{Z}\right\}$,

(ii) $C(\tilde{S})=\left\{\left\langle k, \gamma_{\tilde{S}}(k), \sigma_{\tilde{S}}(k), \mu_{\tilde{S}}(k)\right\rangle: k \in \mathscr{Z}\right\}$,

(iii) $C(\tilde{S})=\left\{\left\langle k, \gamma_{\tilde{S}}(k), 1-\sigma_{\tilde{S}}(k), \mu_{\tilde{S}}(k)\right\rangle: k \in \mathscr{Z}\right\}$.

Neutrosophic sets $(N S s) 0_{N}$ and $1_{N}$ [14] in $\mathscr{Z}$ are introduced as follows:

$1-0_{N}$ can be defined as four types:

(i) $0_{N}=\{\langle k, 0,0,1\rangle: k \in \mathscr{Z}\}$,

(ii) $0_{N}=\{\langle k, 0,1,1\rangle: k \in \mathscr{Z}\}$,

(iii) $0_{N}=\{\langle k, 0,1,0\rangle: k \in \mathscr{Z}\}$,

(iv) $0_{N}=\{\langle k, 0,0,0\rangle: k \in \mathscr{Z}\}$.

$2-1_{N}$ can be defined as four types:

(i) $1_{N}=\{\langle k, 1,0,0\rangle: k \in \mathscr{Z}\}$,

(ii) $1_{N}=\{\langle k, 1,0,1\rangle: k \in \mathscr{Z}\}$,

(iii) $1_{N}=\{\langle k, 1,1,0\rangle: k \in \mathscr{Z}\}$,

(iv) $1_{N}=\{\langle k, 1,1,1\rangle: k \in \mathscr{Z}\}$.

Definition 3. Let $k$ be a non-empty set, and generalized neutrosophic sets GNSs $\tilde{S}$ and $\tilde{R}$ be in the form $\tilde{S}=\left\{k, \mu_{\tilde{S}}(k), \sigma_{\tilde{S}}(k), \gamma_{\tilde{S}}(k)\right\}, B=\left\{k, \mu_{\tilde{R}}(k), \sigma_{\tilde{R}}(k), \gamma_{\tilde{R}}(k)\right\}$. Then, we may consider two possible definitions for subsets $(\tilde{S} \subseteq \tilde{R})[14]$ :

(i) $\tilde{S} \subseteq B \Leftrightarrow \mu_{\tilde{S}}(k) \leq \mu_{B}(k), \sigma_{\tilde{S}}(k) \geq \sigma_{B}(k)$, and $\gamma_{\tilde{S}}(k) \leq \gamma_{B}(k)$,

(ii) $\tilde{S} \subseteq B \Leftrightarrow \mu_{\tilde{S}}(k) \leq \mu_{B}(k), \sigma_{\tilde{S}}(k) \geq \sigma_{B}(k)$, and $\gamma_{\tilde{S}}(k) \geq \gamma_{B}(k)$.

Definition 4. Let $\left\{\tilde{S}_{j}: j \in J\right\}$ be an arbitrary family of NSs in $\mathscr{Z}$. Then,

(i) $\cap \tilde{S}_{j}$ can defined as two types:

$\cap \tilde{S}_{j}=\left\langle k, \underset{j \in J}{\wedge} \mu_{\tilde{S} j}(k), \underset{j \in J}{\wedge \sigma_{\tilde{S} j}}(k), \underset{j \in J}{\vee} \gamma_{\tilde{S} j}(k)\right\rangle$,

$\cap \tilde{S}_{j}=\left\langle k, \underset{j \in J}{\wedge} \mu_{\tilde{S} j}(k), \underset{j \in J}{\vee} \sigma_{\tilde{S} j}(k), \underset{j \in J}{\vee} \gamma_{\tilde{S} j}(k)\right\rangle$. 
(ii) $\cup \tilde{S}_{j}$ can defined as two types:

$$
\begin{aligned}
& \cup \tilde{S}_{j}=\left\langle k, \underset{j \in J}{\vee} \mu_{\tilde{S} j}(k), \underset{j \in J}{\vee} \sigma_{\tilde{S} j}(k), \underset{j \in J}{\wedge} \gamma_{\tilde{S} j}(k)\right\rangle, \\
& \cup \tilde{S}_{j}=\left\langle k, \underset{j \in J}{\vee} \mu_{\tilde{S} j}(k), \underset{j \in J}{\wedge} \sigma_{\tilde{S} j}(k),{\left.\underset{j \in J}{\wedge} \gamma_{\tilde{S} j}(k)\right\rangle, \text { see [14]. }}^{\prime}\right.
\end{aligned}
$$

Definition 5. A neutrosophic topology (NT for short) [16] and a non empty set $\mathscr{Z}$ is a family $\Gamma$ of neutrosophic subsets of $\mathscr{Z}$ satisfying the following axioms:

(i) $0_{N}, 1_{N} \in \Gamma$,

(ii) $\tilde{S}_{1} \cap \tilde{S}_{2} \in \Gamma$ for any $\tilde{S}_{1}, \tilde{S}_{2} \in \Gamma$,

(iii) $\cup \tilde{S}_{i} \in \Gamma, \forall\left\{\tilde{S}_{i} \mid j \in J\right\} \subseteq \Gamma$.

In this case, the pair $(\mathscr{Z}, \Gamma)$ is called a neutrosophic topological space (NTS for short) and any neutrosophic set in $\Gamma$ is known as neutrosophic open set NOS $\in \mathscr{Z}$. The elements of $\Gamma$ are called neutrosophic open sets. A closed neutrosophic set $\tilde{R}$ if and only if its $C(\tilde{R})$ is neutrosophic open.

Note that, for any NTS $\tilde{S}$ in $(\mathscr{Z}, \Gamma)$, we have $\operatorname{NCl}\left(\tilde{S}^{c}\right)=[\operatorname{NInt}(\tilde{S})]^{c}$ and $\operatorname{NInt}\left(\tilde{S}^{c}\right)=[\operatorname{NCl}(\tilde{S})]^{c}$.

Definition 6. Let $\tilde{S}=\left\{\mu_{\tilde{S}}(k), \sigma_{\tilde{S}}(k), \gamma_{\tilde{S}}(k)\right\}$ be a neutrosophic open set and $B=\left\{\mu_{B}(k), \sigma_{B}(k), \gamma_{B}(k)\right\}$ a neutrosophic set on a neutrosophic topological space $(\mathscr{Z}, \Gamma)$. Then,

(i) $\tilde{S}$ is called neutrosophic regular open [14] iff $\tilde{S}=\operatorname{NInt}(\operatorname{NCl}(\tilde{S}))$.

(ii) If $B \in \operatorname{NCS}(\mathscr{Z})$, then $B$ is called neutrosophic regular closed [14] iff $\tilde{S}=\operatorname{NCl}(\operatorname{NInt}(\tilde{S}))$.

Definition 7. Let $(k, \Gamma)$ be NT and $\tilde{S}=\left\{k, \mu_{\tilde{S}}(k), \sigma_{\tilde{S}}(k), \gamma_{\tilde{S}}(k)\right\}$ an NS in $\mathscr{Z}$. Then,

(i) $\operatorname{NCL}(\tilde{S})=\cap\{U: U$ is an NCS in $\mathscr{Z}, \tilde{S} \subseteq U\}$,

(ii) $\operatorname{NInt}(\tilde{S})=\cup\{V: V$ is an NOS in $\mathscr{Z}, V \subseteq \tilde{S}\}$, see [14].

It can be also shown that $\operatorname{NCl}(\tilde{S})$ is an NCS and NInt $(\tilde{S})$ is an NOS in $\mathscr{Z}$. We have

(i) $\tilde{S}$ is in $\mathscr{Z}$ iff $\mathrm{NCl}(\tilde{S})$.

(ii) $\tilde{S}$ is an $N C S$ in $\mathscr{Z}$ iff $N \operatorname{Int}(\tilde{S})=\tilde{S}$.

Definition 8. Let $\tilde{S}$ be an NS and $(\mathscr{Z}, \Gamma)$ an NT. Then,

(i) Neutrosophic semiopen set (NSOS) [12] if $\tilde{S} \subseteq \operatorname{NCl}(\operatorname{NInt}(\tilde{S}))$,

(ii) Neutrosophic preopen set (NPOS) [12] if $\tilde{S} \subseteq \operatorname{NInt}(\operatorname{NNCl}(\tilde{S}))$,

(iii) Neutrosophic $\alpha$-open set $(N \alpha O S)$ [12] if $\tilde{S} \subseteq \operatorname{NInt}(\operatorname{NNCl}(\operatorname{NInt}(\tilde{S})))$,

(iv) Neutrosophic $\beta$-open set $(N \beta O S)$ [12] if $\tilde{S} \subseteq \operatorname{NNCl}(\operatorname{NInt}(\operatorname{NCl}(\tilde{S})))$.

The complement of $\tilde{S}$ is an NSOS, N $\alpha$ OS, NPOS, and NROS, which is called NSCS, N $\alpha$ CS, NPCS, and NRCS, resp.

Definition 9. Let $\tilde{S}=\left\{\tilde{S}_{1}, \tilde{S}_{2}, \tilde{S}_{3}\right\}$ be an NS and $(\mathscr{Z}, \Gamma)$ an NT. Then, the $*$-neutrosophic closure of $\tilde{S}$ $(*-\operatorname{NCl}(\tilde{S})$ for short [12]) and $*$-neutrosophic interior $(*-\operatorname{NInt}(\tilde{S})$ for short [12]) of $\tilde{S}$ are defined by

(i) $\alpha \operatorname{NCl}(\tilde{S})=\cap\{V: V$ is an NRC in $\mathscr{Z}, \tilde{S} \subseteq V\}$,

(ii) $\alpha \operatorname{NInt}(\tilde{S})=\cup\{U: U$ is an NRO in $\mathscr{Z}, U \subseteq \tilde{S}\}$,

(iii) $\operatorname{pNCl}(\tilde{S})=\cap\{V: V$ is an NPC in $\mathscr{Z}, \tilde{S} \subseteq V\}$,

(iv) $p \operatorname{Nint}(\tilde{S})=\cup\{U: U$ is an NPO in $\mathscr{Z}, U \subseteq \tilde{S}\}$,

(v) $\operatorname{sNCl}(\tilde{S})=\cap\{V: V$ is an NSC in $\mathscr{Z}, \tilde{S} \subseteq V\}$,

(vi) $\operatorname{sNInt}(\tilde{S})=\cup\{U: U$ is an NSO in $\mathscr{Z}, U \subseteq \tilde{S}\}$,

(vii) $\beta \operatorname{NCl}(\tilde{S})=\cap\{V: V$ is an $N C \beta C$ in $\mathscr{Z}, \tilde{S} \subseteq V\}$,

(viii) $\beta N \operatorname{Int}(\tilde{S})=\cup\{U: U$ is a $N \beta O$ in $\mathscr{Z}, U \subseteq \tilde{S}\}$,

(ix) $\operatorname{rNCl}(\tilde{S})=\cap\{V: V$ is an NRC in $\mathscr{Z}, \tilde{S} \subseteq V\}$,

(x) $r \operatorname{NInt}(\tilde{S})=\cup\{U: U$ is an NRO in $\mathscr{Z}, U \subseteq \tilde{S}\}$. 
Definition 10. An (NS) $\tilde{S}$ of an NT $(\mathscr{Z}, \Gamma)$ is called a generalized neutrosophic closed set [17] (GNC in short) if $\operatorname{NCl}(\tilde{S}) \subseteq \tilde{B}$ wherever $\tilde{S} \subset \tilde{B}$ and $\tilde{B}$ is a neutrosophic closed set in $\mathscr{Z}$.

Definition 11. An NS $\tilde{S}$ in an NT $\mathscr{Z}$ is said to be a neutrosophic a generalized closed set (NagCS [18]) if $\operatorname{N\alpha NCl}(\tilde{S}) \subseteq \tilde{B}$ whensoever $\tilde{S} \subseteq \tilde{B}$ and $\tilde{B}$ is an NOS in $\mathscr{Z}$. The complement $C(\tilde{S})$ of an NagCS $\tilde{S}$ is an NagOS in $\mathscr{Z}$.

\section{Neutrosophic Generalized Connected Spaces, Neutrosophic Generalized Compact Spaces and Generalized Neutrosophic Extremally Disconnected Spaces}

Definition 12. Let $(\mathscr{Z}, \Gamma)$ and $\left(\mathscr{K}, \Gamma_{1}\right)$ be any two neutrosophic topological spaces.

(i) A function $g:(\mathscr{Z}, \Gamma) \longrightarrow\left(\mathscr{K}, \Gamma_{1}\right)$ is called generalized neutrosophic continuous( $G N$-continuous) $g^{-1}$ of every closed set in $\left(\mathscr{Z}, \Gamma_{1}\right)$ is $G N$-closed in $(\mathscr{Z}, \Gamma)$.

Equivalently, if the inverse image of every open set in $\left(\mathscr{Z}, \Gamma_{1}\right)$ is GN-open in $(\mathscr{Z}, \Gamma)$ :

(ii) A function $g:(\mathscr{Z}, \Gamma) \longrightarrow\left(\mathscr{K}, \Gamma_{1}\right)$ is called generalized neutrosophic irresolute $g^{-1}$ of every $G N$-closed set in $\left(\mathscr{Z}, \Gamma_{1}\right)$ is GN-closed in $(\mathscr{Z}, \Gamma)$.

Equivalently $g^{-1}$ of every GN-open set in $\left(\mathscr{Z}, \Gamma_{1}\right)$ is GN-open in $(\mathscr{Z}, \Gamma)$

(iii) A function $g:(\mathscr{Z}, \Gamma) \longrightarrow\left(\mathscr{K}, \Gamma_{1}\right)$ is said to be strongly neutrosophic continuous if $g^{-1}(\tilde{S})$ is both neutrosophic open and neutrosophic closed in $(\mathscr{Z}, \Gamma)$ for each neutrosophic set $\tilde{S}$ in $\left(\mathscr{Z}, \Gamma_{1}\right)$.

(iv) A function $g:(\mathscr{Z}, \Gamma) \longrightarrow\left(\mathscr{K}, \Gamma_{1}\right)$ is said to be strongly $G N$-continuous if the inverse image of every GN-open set in $\left(\mathscr{Z}, \Gamma_{1}\right)$ is neutrosophic open in $(\mathscr{Z}, \Gamma)$, see ([17] for more details).

Definition 13. An NTS $(\mathscr{Z}, \Gamma)$ is said to be neutrosophic- $T_{\frac{1}{2}}\left(N T_{\frac{1}{2}}\right.$ in short) space if every GNC in $\mathscr{Z}$ is an NC in $\mathscr{Z}$.

Definition 14. Let $(\mathscr{Z}, \Gamma)$ be any neutrosophic topological space. $(\mathscr{Z}, \Gamma)$ is said to be generalized neutrosophic disconnected (in shortly GN-disconnected) if there exists a generalized neutrosophic open and generalized neutrosophic closed set $\tilde{R}$ such that $\tilde{R} \neq 0_{N}$ and $\tilde{R} \neq 1_{N} \cdot(\mathscr{Z}, \Gamma)$ is said to be generalized neutrosophic connected if it is not generalized neutrosophic disconnected.

Proposition 1. Every GN-connected space is neutrosophic connected. However, the converse is not true.

Proof. For a $G N$-connected $(\mathscr{Z}, \Gamma)$ space and let $(\mathscr{Z}, \Gamma)$ not be neutrosophic connected. Hence, there exists a proper neutrosophic set, $\tilde{S}=\left\langle\mu_{\tilde{S}}(x), \sigma_{\tilde{S}}(x), \gamma_{\tilde{S}}(x)\right\rangle \tilde{S} \neq 0_{N}, \tilde{S} \neq 1_{N}$, such that $\tilde{S}$ is both neutrosophic open and neutrosophic closed in $(\mathscr{Z}, \Gamma)$. Since every neutrosophic open set is $G N$-open and neutrosophic closed set is $G N$-closed, $\mathscr{Z}$ is not $G N$-connected. Therefore, $(\mathscr{Z}, \Gamma)$ is neutrosophic connected.

Example 1. Let $\mathscr{Z}=\{u, v, w\}$. Define the neutrosophic sets $\tilde{S}, \tilde{R}$ and $\mathscr{Z}$ in $\mathscr{Z}$ as follows: $\tilde{S}=\left\langle x,\left(\frac{a}{0.4}, \frac{b}{0.5}, \frac{c}{0.5}\right),\left(\frac{a}{0.4}, \frac{b}{0.5}, \frac{c}{0.5}\right),\left(\frac{a}{0.5}, \frac{b}{0.5}, \frac{c}{0.5}\right)\right\rangle, \tilde{R}=\left\langle x,\left(\frac{a}{0.7}, \frac{b}{0.6}, \frac{c}{0.5}\right),\left(\frac{a}{0.7}, \frac{b}{0.6}, \frac{c}{0.5}\right),\left(\frac{a}{0.3}, \frac{b}{0.4}, \frac{c}{0.5}\right)\right\rangle$. Then, the family $\Gamma=\left\{0_{N}, 1_{N}, \tilde{S}, \tilde{R}\right\}$ is neutrosophic topology on $\mathscr{Z}$. It is obvious that $(\mathscr{Z}, \Gamma)$ is NTS. Now, $(\mathscr{Z}, \Gamma)$ is neutrosophic connected. However, it is not a GN-connected for $\tilde{Z}=$ $\left\langle x,\left(\frac{a}{0.5}, \frac{b}{0.6}, \frac{c}{0.5}\right),\left(\frac{a}{0.5}, \frac{b}{0.6}, \frac{c}{0.5}\right),\left(\frac{a}{0.5}, \frac{b}{0.6}, \frac{c}{0.5}\right)\right\rangle$ is GN open and GN closed in $(\mathscr{Z}, \Gamma)$.

Theorem 1. Let $(\mathscr{Z}, \Gamma)$ be a neutrosophic $T_{\frac{1}{2}}$ space; then, $(\mathscr{Z}, \Gamma)$ is neutrosophic connected iff $(\mathscr{Z}, \Gamma)$ is GN-connected.

Proof. Suppose that $(\mathscr{Z}, \Gamma)$ is not $G N$-connected, and there exists a neutrosophic set $\tilde{S}$ which is both $G N$-open and $G N$-closed. Since $(\mathscr{Z}, \Gamma)$ is neutrosophic $T_{\frac{1}{2}}, \tilde{S}$ is both neutrosophic open and neutrosophic closed. Hence, $(\mathscr{Z}, \Gamma)$ is $G N$-connected. Conversely, let $(\mathscr{Z}, \Gamma)$ is $G N$-connected. Suppose that $(\mathscr{Z}, \Gamma)$ is not neutrosophic connected, and there exists a neutrosophic set $\tilde{S}$ such that $\tilde{S}$ is both $N C s$ and $N O s \in(\mathscr{Z}, \Gamma)$. 
Since the neutrosophic open set is $G N$-open and the neutrosophic closed set is $G N$-closed, $(\mathscr{Z}, \Gamma)$ is not $G N$-connected. Hence, $(\mathscr{Z}, \Gamma)$ is neutrosophic connected.

Proposition 2. Suppose $(\mathscr{Z}, \Gamma)$ and $\left(\mathscr{K}, \Gamma_{1}\right)$ are any two NTSs. If $g:(\mathscr{Z}, \Gamma) \longrightarrow\left(\mathscr{K}, \Gamma_{1}\right)$ is GN-continuous surjection and $(\mathscr{Z}, \Gamma)$ is $G N$-connected, then $\left(\mathscr{K}, \Gamma_{1}\right)$ is neutrosophic connected.

Proof. Suppose that $\left(\mathscr{K}, \Gamma_{1}\right)$ is not neutrosophic connected, such that the neutrosophic set $\tilde{S}$ is both neutrosophic open and neutrosophic closed in $\left(\mathscr{K}, \Gamma_{1}\right)$. Since $g$ is $G N$-continuous, $g^{-1}(\tilde{S})$ is $G N$-open and $G N$-closed in $\left((\mathscr{K}, \Gamma)\right.$. Thus, $(\mathscr{K}, \Gamma)$ is not $G N$ connected. Hence, $\left(\mathscr{K}, \Gamma_{1}\right)$ is neutrosophic connected.

Definition 15. Let $(\mathscr{K}, \Gamma)$ be an NT. If a family $\left\{\left\langle k, \mu_{G_{i}}(k), \sigma_{G_{i}}(k), \gamma_{G_{i}}(k): i \in J\right\rangle\right\}$ of $G N$ open sets in $(\mathscr{K}, \Gamma)$ satisfies the condition $\bigcup\left\{\left\langle k, \mu_{G_{i}}(k), \sigma_{G_{i}}(k), \gamma_{G_{i}}(k): i \in J\right\rangle\right\}=1_{N}$, then it is called a GN open cover of $(\mathscr{K}, \Gamma)$. A finite subfamily of a $G N$ open cover $\left\{\left\langle k, \mu_{G_{i}}(k), \sigma_{G_{i}}(k), \gamma_{G_{i}}(k): i \in J\right\rangle\right\}$ of $(\mathscr{Z}, \Gamma)$, which is also a $G N$ open cover of $(\mathscr{K}, \Gamma)$ is called a finite subcover of

$$
\left\{\left\langle k, \mu_{G_{i}}(k), \sigma_{G_{i}}(k), \gamma_{G_{i}}(k): i \in J\right\rangle\right\} .
$$

Definition 16. An NT $(\mathscr{K}, \Gamma)$ is called $G N$ compact iff every $G N$ open cover of $(\mathscr{K}, \Gamma)$ has a finite subcover.

Theorem 2. Let $(\mathscr{K}, \Gamma)$ and $\left(\mathscr{K}, \Gamma_{1}\right)$ be any two NTs, and $g:(\mathscr{Z}, \Gamma) \longrightarrow\left(\mathscr{K}, \Gamma_{1}\right)$ be GN continuous surjection. If $(\mathscr{K}, \Gamma)$ is $G N$-compact, hence so is $\left(\mathscr{K}, \Gamma_{1}\right)$.

Proof. Let $G_{i}=\left\{\left\langle y, \mu_{G_{i}}(x), \sigma_{G_{i}}(x), \gamma_{G_{i}}(x): i \in J\right\rangle\right\}$ be a neutrosophic open cover in $\left(\mathscr{K}, \Gamma_{1}\right)$ with

$$
\widetilde{\bigcup}\left\{\left\langle y, \mu_{G_{i}}(x), \sigma_{G_{i}}(x), \gamma_{G_{i}}(x): i \in J\right\rangle\right\}=\widetilde{\bigcup_{i \in J}} G_{i}=1_{N}
$$

Since $g$ is $G N$ continuous, $g^{-1}\left(G_{i}\right)=G_{i}=\left\{\left\langle y, \mu_{g^{-1}\left(G_{i}\right)}(x), \sigma_{g^{-1}\left(G_{i}\right)}(x), \gamma_{g^{-1}\left(G_{i}\right)}(x): i \in J\right\rangle\right\}$ is GN open cover of $(\mathscr{K}, \Gamma)$. Now,

$$
\widetilde{\bigcup_{i \in J}} g^{-1}\left(G_{i}\right)=g^{-1}\left(\widetilde{\bigcup_{i \in J}} G_{i}\right)=1_{N}
$$

Since $(\mathscr{K}, \Gamma)$ is $G N$ compact, there exists a finite subcover $J_{0} \subset J$, such that

$$
\widetilde{\bigcup_{i \in J_{0}}} g^{-1}\left(G_{i}\right)=1_{N}
$$

Hence,

$$
g\left(\widetilde{\bigcup_{i \in J_{0}}} g^{-1}\left(G_{i}\right)=1_{N}\right), g^{-1}\left(\widetilde{\bigcup} \widetilde{\bigcup}_{i \in J_{0}}\left(G_{i}\right)=1_{N}\right)
$$

That is,

$$
\widetilde{\bigcup_{i \in J_{0}}}\left(G_{i}\right)=1_{N}
$$

Therefore, $\left(\mathscr{K}, \Gamma_{1}\right)$ is neutrosophic compact.

Definition 17. Let $(\mathscr{K}, \Gamma)$ be an $N T$ and $K$ be a neutrosophic set in $(\mathscr{Z}, \Gamma)$. If a family $\left\{\left\langle k, \mu_{G_{i}}(k), \sigma_{G_{i}}(k), \gamma_{G_{i}}(k): i \in J\right\rangle\right\}$ of $G N$ open sets in $(\mathscr{K}, \Gamma)$ satisfies the condition $K \subseteq$ $\bigcup\left\{\left\langle k, \mu_{G_{i}}(k), \sigma_{G_{i}}(k), \gamma_{G_{i}}(k): i \in J\right\rangle\right\}=1_{N}$, then it is called a GN open cover of $K$. A finite subfamily of a GN open cover $\left\{\left\langle k, \mu_{G_{i}}(k), \sigma_{G_{i}}(k), \gamma_{G_{i}}(k): i \in J\right\rangle\right\}$ of $K$, which is also a $G N$ open cover of $K$ is called a finite subcover of $\left\{\left\langle k, \mu_{G_{i}}(k), \sigma_{G_{i}}(k), \gamma_{G_{i}}(k): i \in J\right\rangle\right\}$. 
Definition 18. An NT $(\mathscr{K}, \Gamma)$ is called GN compact iff every GN open cover of $K$ has a finite subcover.

Theorem 3. Let $(\mathscr{K}, \Gamma)$ and $\left(\mathscr{K}, \Gamma_{1}\right)$ be any two NTs, and $g:(\mathscr{Z}, \Gamma) \longrightarrow\left(\mathscr{K}, \Gamma_{1}\right)$ be an $G N$ continuous function. If $K$ is $G N$-compact, then so is $g(K)$ in $\left(\mathscr{K}, \Gamma_{1}\right)$.

Proof. Let $G_{i}=\left\{\left\langle y, \mu_{G_{i}}(x), \sigma_{G_{i}}(x), \gamma_{G_{i}}(x): i \in J\right\rangle\right\}$ be a neutrosophicopen cover of $g(K)$ in $\left(\mathscr{K}, \Gamma_{1}\right)$. That is,

$$
g(K) \subseteq \widetilde{\bigcup_{i \in J}} G_{i}
$$

Since $g$ is $G N$ continuous, $g^{-1}\left(G_{i}\right)=\left\{\left\langle x, \mu_{g^{-1}\left(G_{i}\right)}(x), \sigma_{g^{-1}\left(G_{i}\right)}(x), \gamma_{g^{-1}\left(G_{i}\right)}(x): i \in J\right\rangle\right\}$ is $G N$ open cover of $K$ in $(\mathscr{Z}, \Gamma)$. Now,

$$
K \subseteq g^{-1}\left(\widetilde{\bigcup_{i \in J}} G_{i}\right) \subseteq \widetilde{\bigcup_{i \in J}} g^{-1}\left(G_{i}\right)
$$

Since $K$ is $(\mathscr{Z}, \Gamma)$ is $G N$ compact, there exists a finite subcover $J_{0} \subset J$, such that

$$
K \subseteq \widetilde{\bigcup_{i \in J_{0}}} g^{-1}\left(G_{i}\right)=1_{N}
$$

Hence,

$$
g(K) \subseteq g\left(\widetilde{\bigcup} \widetilde{\bigcup}_{i \in J_{0}} g^{-1}\left(G_{i}\right)\right) \widetilde{\bigcup} \widetilde{U}_{i \in J_{0}}\left(G_{i}\right)
$$

Therefore, $g(K)$ is neutrosophic compact.

Proposition 3. Let $(\mathscr{Z}, \Gamma)$ be a neutrosophic compact space and suppose that $K$ is a $G N$-closed set of $(\mathscr{Z}, \Gamma)$. Then, $K$ is a neutrosophic compact set.

Proof. Let $K_{j}==\left\{\left\langle y, \mu_{G_{i}}(x), \sigma_{G_{i}}(x), \gamma_{G_{i}}(x): i \in J\right\rangle\right\}$ be a family of neutrosophic open set in $(\mathscr{Z}, \Gamma)$ such that

$$
K \subseteq \widetilde{\bigcup_{i \in J}} K_{j}
$$

Since $K$ is $G N$-closed, $N C l(K) \subseteq \widetilde{U}_{i \in J} K_{j}$. Since $(\mathscr{Z}, \Gamma)$ is a neutrosophic compact space, there exists a finite subcover $J_{0} \subseteq J$. Now, $N C l(K) \subseteq \widetilde{U}_{i \in J_{0}} K_{j}$. Hence, $K \subseteq \operatorname{NCl}(K) \subseteq \widetilde{U}_{i \in J_{0}} K_{j}$. Therefore, $K$ is a neutrosophic compact set.

Definition 19. Let $(\mathscr{Z}, \Gamma)$ be any neutrosophic topological space. $(\mathscr{Z}, \Gamma)$ is said to be GN extremally disconnected if $\mathrm{NCl}(\mathrm{K})$ neutrosophic open and $\mathrm{K}$ is GN open.

Proposition 4. For any neutrosophic topological space $(\mathscr{Z}, \Gamma)$, the following are equivalent:

(i) $(\mathscr{Z}, \Gamma)$ is GN extremally disconnected.

(ii) For each GN closed set K, NGNInt ( $\tilde{S})$ is a GN closed set.

(iii) For each $G N$ open set $K$, we have $N G N C l(K)+N G N C l(1-N G N C l(\tilde{S}))=1$.

(iv) For each pair of $G N$ open sets $K$ and $M$ in $(\mathscr{Z}, \Gamma), N G N C l(K)+M=1$, we have $\operatorname{NGNCl}(K)+$ $\operatorname{NGNCl}(B)=1$.

\section{Generalized Neutrosophic Pre-Closed Set}

Definition 20. An NS $\tilde{S}$ is said to be a neutrosophic generalized pre-closed set (GNPCS in short) in $(\mathscr{Z}, \Gamma)$ if $p \operatorname{NCl}(\tilde{S}) \subseteq \tilde{B}$ whensoever $\tilde{S} \subseteq \tilde{B}$ and $\tilde{B}$ is an NO in $\mathscr{Z}$. The family of all $\operatorname{GNPCSs}$ of an NT $(\mathscr{Z}, \Gamma)$ is defined by $\operatorname{GNPC}(\mathscr{Z})$. 
Example 2. Let $\mathscr{Z}=\{a, b\}$ and $\Gamma=\left\{0_{N}, 1_{N}, T\right\}$ be a neutrosophic topology on $\mathscr{Z}$, where $T=$ $\langle(0.2,0.3,0.5),(0.8,0.7,0.7)\rangle$. Then, the NS $\tilde{S}=\langle(0.2,0.2,0.2),(0.8,0.7,0.7)\rangle$ is GNPCs $\in \mathscr{Z}$.

Theorem 4. Every NC is a GNPC, but the converse is not true.

Proof. Let $\tilde{S}$ be an $N C$ in $\mathscr{Z}, \tilde{S} \subseteq \tilde{B}$ and $\tilde{B}$ is NOS in $(\mathscr{Z}, \Gamma)$. Since $p \operatorname{NCl}(\tilde{S}) \subseteq N C l(\tilde{S})$ and $\tilde{S}$ is $N C S$ in $\mathscr{Z}, \operatorname{pNCl}(\tilde{S}) \subseteq \operatorname{NCl}(\tilde{S})=\tilde{S} \subseteq \tilde{B}$. Therefore, $\tilde{S}$ is $G N P C s \in \mathscr{Z}$.

Example 3. Let $\mathscr{Z}=\{u, v\}$ and $\Gamma=\left\{0_{N}, 1_{N}, H\right\}$ be a neutrosophic topology on $\mathscr{Z}$, where $H=$ $\langle(0.2,0.3,0.5),(0.8,0.7,0.7)\rangle$. Then, the NS $\tilde{S}=\langle(0.2,0.2,0.2),(0.8,0.7,0.7)\rangle$ is a GNPC in $\mathscr{Z}$ but not an NCS $\in \mathscr{Z}$.

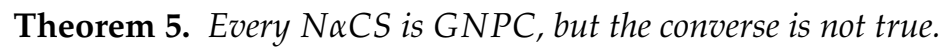

Proof. Let $\tilde{S}$ be an $N \alpha C S$ in $\mathscr{Z}$ and let $\tilde{S} \subseteq \tilde{B}$ and $\tilde{B}$ is an NOS in $(\mathscr{Z}, \Gamma)$. Now, $N C l(N \operatorname{Int}(N C l(\tilde{S}))) \subseteq$ $\tilde{S}$. Since $\tilde{S} \subseteq \operatorname{NCl}(\tilde{S}), \operatorname{NCl}(\operatorname{NInt}(\tilde{S})) \subseteq \operatorname{NCl}(\operatorname{NInt}(\operatorname{NCl}(\tilde{S}))) \subseteq \tilde{S}$. Hence, $p \operatorname{NCl}(\tilde{S}) \subseteq \tilde{S} \subseteq \tilde{B}$. Therefore, $\tilde{S}$ is $G N P C s \in \mathscr{Z}$.

Example 4. Let $\mathscr{Z}=\{u, v\}$ and let $\Gamma=\left\{0_{N}, 1_{N}, H\right\}$ is a neutrosophic topology on $\mathscr{Z}$, where $H=$ $\langle(0.4,0.2,0.5),(0.6,0.7,0.6)\rangle$. Then, the NS $\tilde{S}=\langle(0.3,0.1,0.4),(0.7,0.8,0.7)\rangle$ is a GNPC in $\mathscr{Z}$ but not $\mathrm{N} \alpha \mathrm{Cs}$ in $\mathscr{Z}$ since $\mathrm{NCl}(\operatorname{NInt}(\mathrm{NCl}(\tilde{S})))=\langle(0.5,0.6,0.5),(0.5,0.3,0.6)\rangle \not \subset \tilde{S}$.

Theorem 6. Every $G N \alpha C$ is a GNPC, but the converse is not true.

Proof. Let $\tilde{S}$ be $G N \alpha C s \in \mathscr{Z}, \tilde{S} \subseteq \tilde{B}, \tilde{B}$ be an NOs in $(\mathscr{Z}, \Gamma)$. By Definition 6, $\tilde{S} \cup \operatorname{NCl}(\operatorname{NInt}(\operatorname{NCl}(\tilde{S}))) \subseteq \tilde{B}$. This implies $\operatorname{NCl}(\operatorname{NInt}(\operatorname{NCl}(\tilde{S}))) \subseteq \tilde{B}$ and $\operatorname{NCl}(\operatorname{NInt}(\tilde{S})) \subseteq \tilde{B}$. Therefore, $\operatorname{pNCl}(\tilde{S})=\tilde{S} \cup$ $\operatorname{NCl}(\operatorname{NInt}(\tilde{S})) \subseteq \tilde{B}$. Hence, $\tilde{S}$ is $G N P C s \in \mathscr{Z}$.

Example 5. Let $\mathscr{Z}=\{u, v\}$ and $\Gamma=\left\{0_{N}, 1_{N}, H\right\}$ be a neutrosophic topology on $\mathscr{Z}$, where $H=$ $\langle(0.5,0.6,0.6),(0.5,0.4,0.4)\rangle$. Then, the NS $\tilde{S}=\langle(0.4,0.5,0.5),(0.6,0.5,0.5)\rangle$ is GNPC in $\mathscr{Z}$ but not $\mathrm{GN} \alpha \mathrm{C}$ in $\mathscr{Z}$ since $\alpha \mathrm{NCl}(\tilde{S})=1_{N} \not \subset H$.

Definition 21. An NS $\tilde{S}$ is said to be a neutrosophic generalized pre-closed set (GNSCS) in $(\mathscr{Z}, \Gamma)$ if $\operatorname{SNCl}(\tilde{S}) \subseteq \tilde{B}$ whensoever $\tilde{S} \subseteq \tilde{B}$ and $\tilde{B}$ is an NO in $\mathscr{Z}$. The family of all GNSCSs of an NT $(\mathscr{Z}, \Gamma)$ is defined by $\operatorname{GNSC}(\mathscr{Z})$.

Proposition 5. Let $\tilde{S}, B$ be a two GNPCs of an NT $(\mathscr{Z}, \Gamma)$. NGSC and NGPC are independent.

Example 6. Let $\mathscr{Z}=\{u, v\}, \Gamma=\left\{0_{N}, 1_{N}, H\right\}$ be a neutrosophic topology on $\mathscr{Z}$, where $H=$ $\langle(0.5,0.4,0.4),(0.5,0.6,0.5)\rangle$. Then, the NS $\tilde{S}=H$ is GNSC but not GNPC in $\mathscr{Z}$ since $\tilde{S} \subseteq H$ but $\operatorname{pNCl}(\tilde{S})=\langle(0.5,0.6,0.4),(0.5,0.4,0.5)\rangle \not \subset H$

Example 7. Let $\mathscr{Z}=\{u, v\}, \Gamma=\left\{0_{N}, 1_{N}, H\right\}$ be a neutrosophic topology on $\mathscr{Z}$, where $H=$ $\langle(0.7,0.9,0.7),(0.3,0.1,0.1)\rangle$. Then, the NS $\tilde{S}=\langle(0.6,0.7,0.6),(0.4,0.3,0.4)\rangle$ is GNPC but not GNsC in $\mathscr{Z}$ since $\operatorname{sNCl}(\tilde{S})=1_{N} \subseteq H$.

Proposition 6. NSC and GNPC are independent.

Example 8. Let $\mathscr{Z}=\{a, b\}, \Gamma=\left\{0_{N}, 1_{N}, T\right\}$ be a neutrosophic topology on $\mathscr{Z}$, where $T=$ $\langle(0.5,0.2,0.3),(0.5,0.6,0.5)\rangle$. Then, the NS $\tilde{S}=T$ is an NSC but not GNPC in $\mathscr{Z}$ since $\tilde{S} \subseteq T$ but $\operatorname{pNCl}(\tilde{S})=1\langle(0.5,0.6,0.5),(0.5,0.2,0.3)\rangle \not \subset T$. 
Example 9. Let $\mathscr{Z}=\{u, v\}, \Gamma=\left\{0_{N}, 1_{N}, H\right\}$ be a neutrosophic topology on $\mathscr{Z}$, where $H=\langle(0.8,0.8,0.8),(0.2,0.2,0.2)\rangle$. Then, the NS $\tilde{S}=\langle(0.8,0.8,0.8),(0.2,0.2,0.2)\rangle$ is GNPC but not an $N S C$ in $\mathscr{Z}$ since $\operatorname{Nint}(\operatorname{NCl}(\tilde{S})) \not \subset \tilde{S}$.

The following Figure 1 shows the implication relations between GNPC set and the other existed ones.

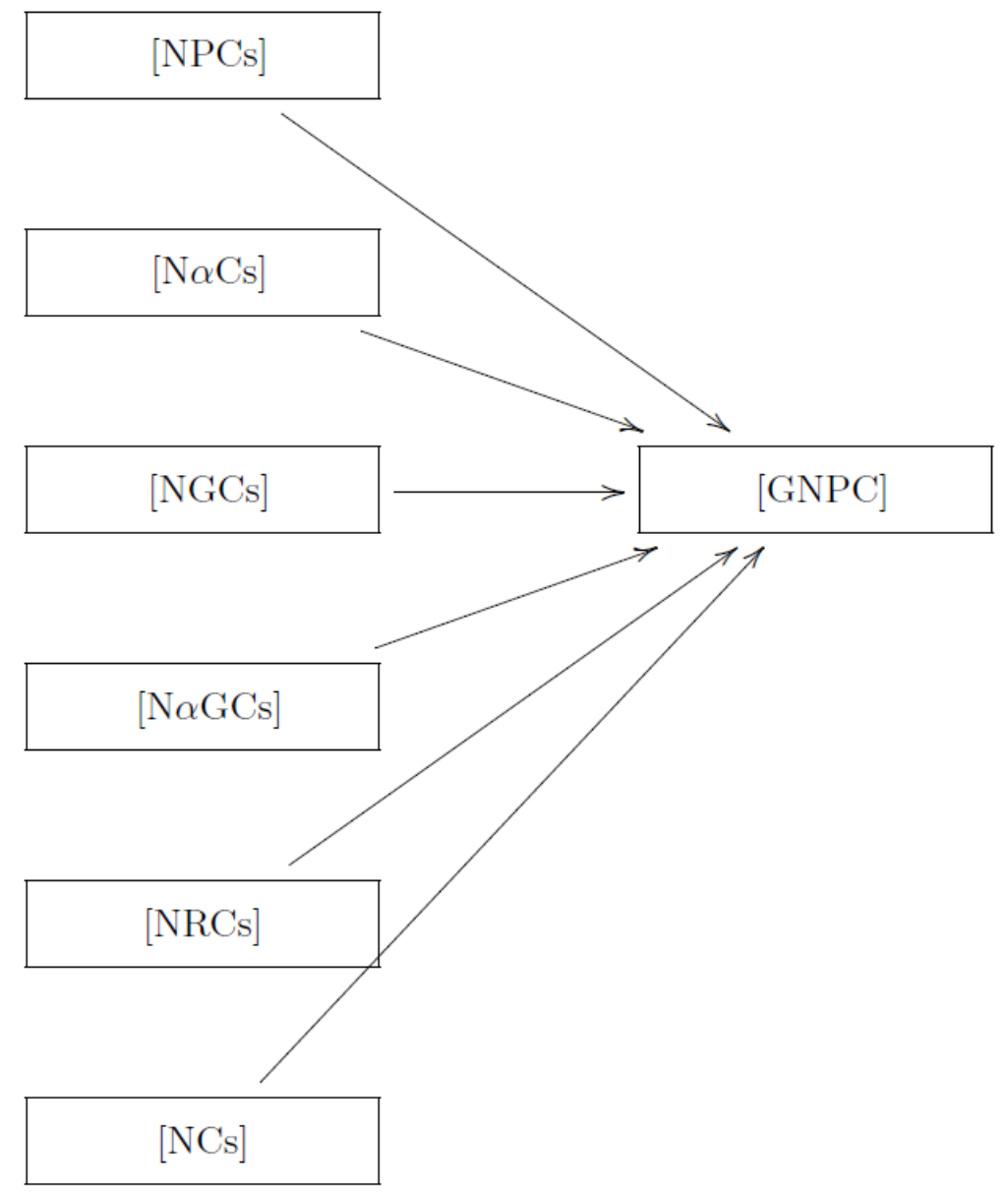

Figure 1. Relation between GNPC and others exists set.

Remark 1. Let $\tilde{S}, B$ be a two GNPCs of an NT ( $\mathscr{Z}, \Gamma)$. Then, the union of any two GNPCs is not a GNPC in general-see the following example.

Example 10. Let $(\mathscr{Z}, \Gamma)$ be a neutrosophic topology set on $\mathscr{Z}$, where $\mathscr{Z}=\{u, v\}$, $T=\langle(0.6,0.8,0.6),(0.4,0.2,0.2)\rangle$. Then, $\Gamma=\left\{0_{N}, 1_{N}, T\right\}$ is neutrosophic topology on $\mathscr{Z}$ and the $N S \tilde{S}=\langle(0.2,0.9,0.3),(0.8,0.2,0.6)\rangle, B=\langle(0.6,0.7,0.6),(0.4,0.3,0.4)\rangle$ are GNPCSs but $\tilde{S} \cup B$ is not a GNPC in $\mathscr{Z}$.

\section{Generalized Neutrosophic Pre-Open Sets}

In this section, we present generalized neutrosophic pre-open sets and investigate some of their properties.

Definition 22. An NS $\tilde{S}$ is said to be a generalized neutrosophic pre-open set (GNPOS) in ( $\mathscr{Z}, \Gamma)$ if the complement $\tilde{S}^{c}$ is a GNPCS in $\mathscr{Z}$. The family of all GNPOSs of NTS $(\mathscr{Z}, \Gamma)$ is denoted by GNPO $(\mathscr{Z})$. 
Example 11. Let $\mathscr{Z}=\{u, v\}$ and $\Gamma=\left\{0_{N}, 1_{N}, H\right\}$ be a neutrosophic topology on $\mathscr{Z}$, where $H=\langle(0.8,0.7,0.8),(0.3,0.4,0.3)\rangle$. Then, the NS $\tilde{S}=\langle(0.9,0.8,0.8),(0.3,0.3,0.3)\rangle$ is GNPO $\in \mathscr{Z}$.

Theorem 7. Let $(\mathscr{Z}, \Gamma)$ be an NT. Then, for every $\tilde{S} \in G N P O(\mathscr{Z})$ and for every $\tilde{R} \in N S(\mathscr{Z}), p N \operatorname{Int}(\tilde{S}) \subseteq$ $\tilde{R} \subseteq \tilde{S}$ implies $\tilde{R} \in G N P O(\mathscr{Z})$.

Proof. By Theorem $\tilde{S}^{c} \subseteq \tilde{R}^{c} \subseteq(p \operatorname{NInt}(\tilde{S}))^{c}$. Let $\tilde{R}^{c} \subseteq \tilde{R}$ and $\tilde{R}$ be NOs. Since $\tilde{S}^{c} \subseteq B^{c}, \tilde{S}^{c} \subseteq \tilde{R}$. However, $\tilde{S}^{c}$ is a GNPCs, $p \operatorname{NCl}\left(\tilde{S}^{c}\right) \subseteq \tilde{R}$. In addition, $\tilde{R}^{c} \subseteq(p N \operatorname{Int}(\tilde{S}))^{c}=p \operatorname{NCl}\left(\tilde{S}^{c}\right)$ (by theorem). Therefore, $\operatorname{pNCl}\left(\tilde{R}^{c}\right) \subseteq \operatorname{pNCl}\left(\tilde{S}^{c}\right) \subseteq \tilde{R}$. Hence, $B^{c}$ is GNPC. This implies that $\tilde{R}$ is a GNPO of $\mathscr{Z}$.

Remark 2. Let $\tilde{S}, \tilde{R}$ be two GNPOs of an NT $(\mathscr{Z}, \Gamma)$. The intersection of any two GNPOSs is not a GNPO in general.

Example 12. Let $\mathscr{Z}=\{u, v\}$ and $\Gamma=\left\{0_{N}, 1_{N}, H\right\}$ be a neutrosophic topology on $\mathscr{Z}$, where $H=\langle(0.6,0.8,0.6),(0.4,0.2,0.4)\rangle$. Then, the NSs, $\tilde{S}=\langle(0.9,0.2,0.1),(0.1,0.8,0.2)\rangle$ and $\tilde{R}=$ $\langle(0.4,0.3,0.4),(0.6,0.7,0.6)\rangle$ is $G N P O$, but $\tilde{S} \cap \tilde{R}$ is not $G N P O \in \mathscr{Z}$.

Theorem 8. For any an NTS $(\mathscr{Z}, \Gamma)$, the following hold:

(i) Every NO is GNPO,

(ii) Every NSO is GNPO,

(iii) Every $N \alpha O$ is GNPO,

(iv) Every NPO is GNPO.

Proof. The proof is clear, so it has been omitted.

The converses are not true in general.

Example 13. Let $\mathscr{Z}=\{u, v\}$ and $H=\langle(0.2,0.3,0.2),(0.8,0.7,0.7)\rangle$. Then, $\Gamma=\left\{0_{N}, 1_{N}, H\right\}$ is $a$ neutrosophic topology on $\mathscr{Z}$, an NS $\tilde{S}=\langle(0.8,0.7,0.7),(0.2,0.2,0.2)\rangle$ is an NSO in $(\mathscr{Z}, \Gamma)$ but not an $N O \in \mathscr{Z}$.

Example 14. Let $\mathscr{Z}=\{u, v\}$ and $\Gamma=\left\{0_{N}, 1_{N}, H\right\}$ be neutrosophic topology on $\mathscr{Z}$, where $H=\langle(0.6,0.4,0.7),(0.7,0.4,0.6)\rangle$. Then, an NS $\tilde{S}=\langle(0.2,0.7,0.7),(0.8,0.3,0.8)\rangle$ is GNPO but not an $N S O \in \mathscr{Z}$.

Example 15. Let $\mathscr{Z}=\{u, v\}$ and $\Gamma=\left\{0_{N}, 1_{N}, H\right\}$ be a neutrosophic topology on $\mathscr{Z}$, where $H=\langle(0.4,0.2,0.4),(0.6,0.7,0.6)\rangle$. Then, an NS $\tilde{S}=\langle(0.8,0.9,0.8),(0.4,0.2,0.3)\rangle$ is GNPO but not an $N \alpha O \in \mathscr{Z}$.

Example 16. Let $\mathscr{Z}=\{u, v\}$ and $\Gamma=\left\{0_{N}, 1_{N}, H\right\}$ be a neutrosophic topology on $\mathscr{Z}$, where $H=\langle(0.6,0.5,0.6),(0.5,0.6,0.5)\rangle$. Then, an NS $\tilde{S}=\langle(0.8,0.7,0.8),(0.4,0.5,0.3)\rangle$ is GNPO but not an $N P O \in \mathscr{Z}$.

Theorem 9. Let $(\mathscr{Z}, \Gamma)$ be an $N T$. If $\tilde{S} \in G N P O(\mathscr{Z})$, then $\tilde{R} \subseteq \operatorname{NInt}(N C l(\tilde{S}))$ whensoever $\tilde{R} \subseteq \tilde{S}$ and $\tilde{R}$ is an NC in $\mathscr{Z}$.

Proof. Let $\tilde{S} \in G N P O(\mathscr{Z})$. Then, $\tilde{S}^{c}$ is $G n P C S$ in $\mathscr{Z}$. Therefore, $p N C l\left(\tilde{S}^{c}\right) \subseteq \tilde{B}$ whensoever $\tilde{S}^{c} \subseteq \tilde{B}$ and $\tilde{B}$ is an $N O$ in $\mathscr{Z}$. That is, $N C l\left(\operatorname{NInt}\left(\tilde{S}^{c}\right)\right) \subseteq \tilde{B}$. This implies $\tilde{B}^{c} \subseteq \operatorname{NInt}(\operatorname{NCl}(\tilde{S}))$ whensoever $\tilde{B}^{c} \subseteq \tilde{S}$ and $\tilde{B}^{c}$ is $N C s$ in $\mathscr{Z}$. Replacing $\tilde{B}^{c}$, by $\tilde{R}$, we get $\tilde{R} \subseteq \operatorname{NInt}(\operatorname{NCl}(\tilde{S}))$ whensoever $\tilde{R} \subseteq \tilde{S}$ and $\tilde{R}$ is an NC in $\mathscr{Z}$.

Theorem 10. For NS $\tilde{S}, \tilde{S}$ is an NO and GNPC in $\mathscr{Z}$ if and only if $\tilde{S}$ is an NRO in $\mathscr{Z}$. 
Proof. $\Longrightarrow$ Let $\tilde{S}$ be an $N O$ and a GNPCS in $\mathscr{Z}$. Then, $p N C l(\tilde{S}) \subseteq \tilde{S}$. This implies $N C l(\operatorname{NInt}(\tilde{S})) \subseteq \tilde{S}$. Since $\tilde{S}$ is an $N O$, it is an NPO. Hence, $\tilde{S} \subseteq \operatorname{NInt}(\operatorname{NCl}(\tilde{S}))$. Therefore, $\tilde{S}=\operatorname{NInt}(\operatorname{NCl}(\tilde{S}))$. Hence, $\tilde{S}$ is an $N R O$ in $\mathscr{Z}$.

$\Longleftarrow$ Let $\tilde{S}$ be an $N R O$ in $\mathscr{Z}$. Therefore, $\tilde{S}=\operatorname{NInt}(N C l(\tilde{S}))$. Let $\tilde{S} \subseteq \tilde{B}$ and $\tilde{B}$ be an $N O$ in $\mathscr{Z}$. This implies $p \operatorname{NCl}(\tilde{S}) \subseteq \tilde{S}$. Hence, $\tilde{S}$ is $G N P C$ in $\mathscr{Z}$.

Theorem 11. An NS $\tilde{S}$ of an $N T(\mathscr{Z}, \Gamma)$ is a GNPO iff $H \subseteq p N \operatorname{Int}(\tilde{S})$, whensoever $H$ is an $N C$ and $H \subseteq \tilde{S}$.

Proof. $\Longrightarrow$ Let $\tilde{S}$ be $G N P O$ in $\mathscr{Z}$. Let $H$ be an NCs and $H \subseteq \tilde{S}$. Then, $H^{c}$ is an NOS in $\mathscr{Z}$ such that $\tilde{S}^{c} \subseteq H^{c}$. Since $\tilde{S}^{c}$ is $G N P C$, we have $p \operatorname{NCl}\left(\tilde{S}^{c}\right) \subseteq H^{c}$. Hence, $(p \operatorname{NInt}(\tilde{S}))^{c} \subseteq H^{c}$. Therefore, $H \subseteq p N \operatorname{Int}(\tilde{S})$.

$\Longleftarrow$ Suppose $\tilde{S}$ is an $N S$ of $\mathscr{Z}$ and let $H \subseteq p N \operatorname{Int}(\tilde{S})$ whensoever $H$ is an $N C$ and $H \subseteq \tilde{S}$. Then, $\tilde{S}^{c} \subseteq H^{c}$ and $H^{c}$ is an NO. By assumption, $(p \operatorname{Nint}(\tilde{S}))^{c} \subseteq H^{c}$, which implies $p \operatorname{NCl}\left(\tilde{S}^{c}\right) \subseteq H^{c}$. Therefore, $\tilde{S}^{c}$ is GNPCS of $\mathscr{Z}$. Hence, $\tilde{S}$ is a GNPOS of $\mathscr{Z}$.

Corollary 1. An NS $\tilde{S}$ of an NTS $(\mathscr{Z}, \Gamma)$ is GNPO iff $H \subseteq N \operatorname{Nint}(N C l(\tilde{S}))$, whensoever $H$ is an NC and $H \subseteq \tilde{S}$.

Proof. $\Longrightarrow$ Let $\tilde{S}$ is a GNPOS in $\mathscr{Z}$. Let $H$ be an NCS and $H \subseteq \tilde{S}$. Then, $H^{c}$ is an NOS in $\mathscr{Z}$ such that $\tilde{S}^{c} \subseteq H^{c}$. Since $\tilde{S}^{c}$ is $G N P C$, we have $\operatorname{pNCl}\left(\tilde{S}^{c}\right) \subseteq H^{c}$. Therefore, $\operatorname{NCl}\left(\operatorname{NInt}\left(\tilde{S}^{c}\right)\right) \subseteq H^{c}$. Hence, $(\operatorname{NInt}(\operatorname{NCl}(\tilde{S})))^{c} \subseteq H^{c}$. This implies $H \subseteq \operatorname{NInt}(\operatorname{NCl}(\tilde{S}))$.

$\Longleftarrow$ Suppose $\tilde{S}$ be an $N S$ of $\mathscr{Z}$ and $H \subseteq N \operatorname{Int}(N C l(\tilde{S}))$, whensoever $H$ is an $N C$ and $H \subseteq \tilde{S}$. Then, $\tilde{S}^{c} \subseteq H^{c}$ and $H^{c}$ is an NO. By assumption, $(\operatorname{NInt}(N C l(\tilde{S})))^{c} \subseteq H^{c}$. Hence, $N C l\left(\operatorname{NInt}\left(\tilde{S}^{c}\right)\right) \subseteq H^{c}$. This implies $p N C l\left(\tilde{S}^{c}\right) \subseteq H^{c}$. Hence, $\tilde{S}$ is a GNPOS of $\mathscr{Z}$.

\section{Applications of Generalized Neutrosophic Pre-Closed Sets}

Definition 23. An NTS $(\mathscr{Z}, \Gamma)$ is said to be neutrosophic- $p T_{\frac{1}{2}}\left(N p T_{\frac{1}{2}}\right.$ in short) space if every GNPC in $\mathscr{Z}$ is an $N C s \in \mathscr{Z}$.

Definition 24. An NTS $(\mathscr{Z}, \Gamma)$ is said to be neutrosophic-gp $T_{\frac{1}{2}}\left(N g p T_{\frac{1}{2}}\right.$ in short) space if every GNPC in $\mathscr{Z}$ is an NPCs $\in \mathscr{Z}$.

Theorem 12. Every $N p T_{\frac{1}{2}}$ space is an $N g p T_{\frac{1}{2}}$ space.

Proof. Let $\mathscr{Z}$ be an $N p T_{\frac{1}{2}}$ space and $\tilde{S}$ be GNPC $\in \mathscr{Z}$. By assumption, $\tilde{S}$ is NCs in $\mathscr{Z}$. Since every NC is an $N P C, \tilde{S}$ is an $N P C$ in $\mathscr{Z}$. Hence, $\mathscr{Z}$ is an $N g p T_{\frac{1}{2}}$ space.

The converse is not true.

Example 17. Let $\mathscr{Z}=\{u, v\}, H=\langle(0.9,0.9,0.9),(0.1,0.1,0.1)\rangle$ and $\Gamma=\left\{0_{N}, 1_{N}, H\right\}$. Then, $(\mathscr{Z}, \Gamma)$ is an $N g p T_{\frac{1}{2}}$ space, but it is not $N p T_{\frac{1}{2}}$ since an $N S H=\langle(0.2,0.3,0.3),(0.8,0.7,0.7)\rangle$ is GNPC but not an NCS $\in \mathscr{Z}^{2}$.

Theorem 13. Let $(\mathscr{Z}, \Gamma)$ be an $N T$ and $\mathscr{Z}$ is an $N p T_{\frac{1}{2}}$ space; then,

(i) the union of GNPCs is GNPC,

(ii) the intersection of GNPOs is GNPO.

Proof. (i) Let $\left\{\tilde{S}_{i}\right\}_{i \in J}$ be a collection of GNPCs in an $N p T_{\frac{1}{2}}$ space $(\mathscr{Z}, \Gamma)$. Thus, every GNPCs is an NCS. However, the union of an NC is an NCS. Therefore, the Union of GNPCs is GNPCs in $\mathscr{Z}$.

(ii) Proved by taking complement in (i). 
Theorem 14. An NT $\mathscr{Z}$ is an $N g p T_{\frac{1}{2}}$ space iff $G N P O(\mathscr{Z})=N P O(\mathscr{Z})$.

Proof. $\Longrightarrow$ Let $\tilde{S}$ be a GNPOs in $\mathscr{Z}$; then, $\tilde{S}^{c}$ is GNPCs in $\mathscr{Z}$. By assumption, $\tilde{S}^{c}$ is an NPCs in $\mathscr{Z}$. Thus, $\tilde{S}$ is NPOs in $\mathscr{Z}$. Hence, $G N P O(\mathscr{Z})=N P O(\mathscr{Z})$.

$\Longleftarrow$ Let $\tilde{S}$ be $G N P C \in \mathscr{Z}$. Then, $\tilde{S}^{c}$ is GNPO in $\mathscr{Z}$. By assumption, $\tilde{S}^{c}$ is an NPO in $\mathscr{Z}$. Thus, $\tilde{S}$ is an $N P C \in \mathscr{Z}$. Therefore, $\mathscr{Z}$ is an $N g p T_{\frac{1}{2}}$ space.

Theorem 15. For an NTS $(\mathscr{Z}, \Gamma)$, the following are equivalent:

(i) $(\mathscr{Z}, \Gamma)$ is a neutrosophic pre- $T_{1}$ space.

(ii) Every non-empty set of $\mathscr{Z}$ is either an NPCS or NPOS.

Proof. $(i) \Longrightarrow(i i)$. Suppose that $(\mathscr{Z}, \Gamma)$ is a neutrosophic pre- $T_{\frac{1}{2}}$ space. Suppose that $\{x\}$ is not an NPCS for some $x \in \mathscr{Z}$. Then, $\mathscr{Z}-\{x\}$ is not an NPOS and hence $\mathscr{Z}$ is the only an NPOS containing $\mathscr{Z}-\{x\}$. Hence, $\mathscr{Z}-\{x\}$ is an NPGCS in $(\mathscr{Z}, \Gamma)$. Since $(\mathscr{Z}, \Gamma)$ is a neutrosophic pre- $T_{\frac{1}{2}}$ space, then $\mathscr{Z}-\{x\}$ is an NPCS or equivalently $\{x\}$ is an NPOS. (ii) $\Longrightarrow(i)$. Let every singleton set of $\mathscr{Z}$ be either NPCS or NPOS. Let $\tilde{S}$ be an NPGCS of $(\mathscr{Z}, \Gamma)$. Let $x \in \mathscr{Z}$. We show that $x \in \mathscr{Z}$ in two cases.

Case (i): Suppose that $\{x\}$ is NPCS. If $x \notin \tilde{S}$, then $x \in p N C l(\tilde{S})-\tilde{S}$. Now, $p N C l(\tilde{S})-\tilde{S}$ contains a non-empty NPCS. Since $\tilde{S}$ is NPGCS, by Theorem 7, we arrived to a contradiction. Hence, $x \in \mathscr{Z}$.

Case (ii): Let $\{x\}$ be NPOS. Since $x \in p \operatorname{NCl}(\tilde{S})$, then $\{x\} \cap \tilde{S} \neq \phi$. Thus, $x \in \mathscr{Z}$. Thus, in any case $x \in \mathscr{Z}$. Thus, $\operatorname{PNCl}(\tilde{S}) \subseteq \tilde{S}$. Hence, $\tilde{S}=p \operatorname{NCl}(\tilde{S})$ or equivalently $\tilde{S}$ is an NPCS. Thus, every NPGCS is an NCS. Therefore, $(\mathscr{Z}, \Gamma)$ is neutrosophic pre- $T_{\frac{1}{2}}$ space.

\section{Conclusions}

We have introduced generalized neutrosophic pre-closed sets and generalized neutrosophic pre-open sets over neutrosophic topology space. Many results have been established to show how far topological structures are preserved by these neutrosophic pre-closed. We also have provided examples where such properties fail to be preserved. In this paper, we have studied a few ideas only; it will be necessary to carry out more theoretical research to establish a general framework for decision-making and to define patterns for complex network conceiving and practical application.

Author Contributions: All authors have contributed equally to this paper. The individual responsibilities and contribution of all authors can be described as follows: the idea of this paper was put forward by W.A.-O. W.A.-O. completed the preparatory work of the paper. S.J. analyzed the existing work. The revision and submission of this paper was completed by W.A.-O.

Funding: This research received no external funding.

Conflicts of Interest: The authors declare no conflicts of interest.

\section{References}

1. Zadeh, L. Fuzzy sets. Inform Control 1965, 8, 338-353. [CrossRef]

2. Chang, C.L. Fuzzy topological spaces. J. Math. Anal. Appl. 1968, 24, 182-190. [CrossRef]

3. Scellato, S.; Fortuna, L.; Frasca, M.; Gómez-Gardenes, J.; Latora, V. Traffic optimization in transport networks based on local routing. Eur. Phys. J. B 2010, 73, 303-308. [CrossRef]

4. Atanassov, K. Intuitionistic fuzzy sets. In VII ITKR's Session; Publishing House: Sofia, Bulgaria, 1983.

5. Atanassov, K.; Stoeva, S. Intuitionistic L-fuzzy sets. In Cybernetics and System Research; Trappl, R., Ed.; Elsevier: Amsterdam, The Netherlands, 1984; Volume 2, pp. 539-540.

6. Coker, D. An introduction to intuitionistic fuzzy topological space. Fuzzy Sets Syst. 1997, 88, 81-89. [CrossRef]

7. Balasubramanin, G.; Sundaram, P. On some generalizations of fuzzy continuous functions. Fuzzy Sets Syst. 1997, 86, 93-100. [CrossRef] 
8. Smarandache, F. A Unifying Field in Logics: Neutrosophic Logic. Neutrosophy, Neutrosophic Set, Neutrosophic Probability, 3rd ed.; American Research Press: Rehoboth, NM, USA, 1999.

9. Lupianez, F.G. On neutrosophic sets and topology. Procedia Comput. Sci. 2017, 120, 975-982. [CrossRef]

10. Liu, P.; Wang, P. Some q-Rung Orthopair Fuzzy Aggregation Operators and Their Applications to Multiple-Attribute Decision Making. Int. J. Intell. Syst. 2018, 33, 259-280. [CrossRef]

11. Zhou, L. Wu, W. Z. and Zhang, W. X. On characterization of intuitionistic fuzzy rough sets based on intuitionistic fuzzy implicators. Inf. Sci. 2009, 179, 7, 883-898. [CrossRef]

12. Al-Omeri, W.; Smarandache, F. New Neutrosophic Sets via Neutrosophic Topological Spaces. In Neutrosophic Operational Research; Smarandache, F., Pramanik, S., Eds.; Pons Editions: Brussels, Belgium, 2017; Volume I, pp. 189-209.

13. Al-Omeri, W.F. Neutrosophic crisp sets via neutrosophic crisp topological spaces. Neutrosophic Sets Syst. 2016, 13, 96-104.

14. Salama, A.A.; Broumi, S.; Alblowi, S.A. Introduction to neutrosophic topological spatial region, possible application to gis topological rules. Inf. Eng. Electron. Bus. 2014, 6, 15-21. [CrossRef]

15. Salama, A.; Alblowi, S. Generalized neutrosophic set and generalized neutrousophic topological spaces. J. Comput. Sci. Eng. 2012, 2, 29-32.

16. Salama, A.A.; Smarandache, F.; Kroumov, V. Neutrosophic closed set and neutrosophic continuous functions. Neutrosophic Sets Syst. 2014, 4, 4-8.

17. Dhavaseelan, R.; Jafari, S. Generalized neutrosophic closed sets. In New Trends in Neutrosophic Theory and Application; Smarandache, F., Pramanik, S., Eds.; Pons Editions: Brussels, Belgium., 2018, Volume 2, pp. 261-274.

18. Jayanthi, D. Generalized closed sets in neutrosophic topological spaces. Int. J. Math. Trends Technol. Spec. Issue 2018, 10, 88-91.

(C) 2018 by the authors. Licensee MDPI, Basel, Switzerland. This article is an open access article distributed under the terms and conditions of the Creative Commons Attribution (CC BY) license (http://creativecommons.org/licenses/by/4.0/). 